Proceedings of the 42th "Jaszowiec" International School and Conference on the Physics of Semiconductors, Wisła 2013

\title{
Carrier Dynamics and Dynamic Band-Bending in Type-II ZnTe/ZnSe Quantum Dots
}

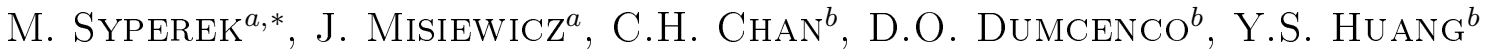 \\ AND W.C. $\mathrm{CHOU}^{c}$ \\ ${ }^{a}$ Institute of Physics, Wrocław University of Technology, Wybrzeże Wyspiańskiego 27, 50-370 Wrocław, Poland \\ ${ }^{b}$ Department of Electronic Engineering, National Taiwan University of Science and Technology, Taipei 106, Taiwan \\ ${ }^{c}$ Department of Electrophysics, National Chiao Tung University, Hsin Chu 300, Taiwan
}

\begin{abstract}
Carrier dynamics and dynamic band-bending in self-assembled ZnTe/ZnSe quantum dots have been studied by means of time-resolved photoluminescence experiment at low temperatures. The experiment reveals clearly type-II character of the confinement potential in the dot manifested in: (i) long photoluminescence decay time constant of 28-35 ns, and (ii) temporal shift of the quantum dot peak emission towards low energy following the laser pulse excitation. The magnitude of the spectral shift $\Delta E$ depends on the dot size and the power density of excitation pulse. For the dots under study and given experimental conditions $\Delta E \approx 28 \div 42 \mathrm{meV}$.
\end{abstract}

DOI: 10.12693 /APhysPolA.124.821

PACS: 78.67.Hc, 78.55.Et, 78.47.J-

\section{Introduction}

Semiconductor quantum dots (QDs) of a type-II band alignment have an additional degree of freedom related to control of the Coulomb interaction between electron and hole within an exciton, interesting from the point of view of tuning of their optical and electronic properties. The internal electric field induced by a spatial separation of an electron and a hole influences several important parameters, including the confined levels energy structure, the transition oscillator strength and thus the radiative lifetime, and the strength of an electron-hole $(\mathrm{e}-\mathrm{h})$ Coulomb interaction. In this context, the type-II ZnTe/ZnSe QD system is quite attractive. Electrons residing in the $\mathrm{ZnSe}$ matrix and holes in the ZnTe island [1], deep confining potential for holes $(\approx 1 \mathrm{eV})$ [1], emission spectrum in the visible spectral range, high electron-hole binding energy of 13-20 meV [2] make such a QDs system interesting not only for foreseen device applications, but also for the study of some basic physical phenomena. The very recent observation of the Aharonov-Bohm effect for electrons with a ring-like wave-function confinement in the type-II ZnTe/ZnSe QDs is one of the most spectacular examples [3]. Although several original papers concerning carrier dynamics in such QDs have been already published [3-6], the influence of peculiar electron and hole confinement on carrier and band structure relaxation is still far from being fully understood.

In this work we present experimental studies on carrier dynamics and dynamic-band bending effect in the type-II ZnTe/ZnSe QDs by means of low-temperature photoluminescence (PL) and time-resolved photoluminescence

*corresponding author; e-mail: marcin.syperek@pwr.wroc.pl
(TRPL). We take advantage of the streak camera detection in the excitation power dependent TRPL experiment to obtain information about the magnitude of the spectral shift related to the band-bending effect in these dots.

\section{Investigated structures and experimental details}

The ZnTe QDs were grown in a molecular beam epitaxy process on a $200 \mathrm{~nm}$ thick ZnSe buffer layer previously deposited on a GaAs substrate. The dots were covered by a $50 \mathrm{~nm}$ thick ZnSe layer [7]. The VolmerWeber growth mode accounts for the self-assembled QD formation. Three QD structures are considered, with the average coverage of 2.5 (QD1), 2.7 (QD2), and 3.0 (QD3) monolayers of ZnTe. Therefore the average dot size increases from QD1 structure to QD3 one with the average dot diameter and height of $\approx 40 \mathrm{~nm}$ and $\approx 7.5 \mathrm{~nm}$, respectively. The surface density of QDs is $\approx 1.5 \times 10^{9} \mathrm{~cm}^{-2}[7]$.

For PL and TRPL experiments the sample was held in a helium close-cycle refrigerator at $T=10 \mathrm{~K}$. In the case of PL and TRPL experiment with the streak camera detection, the QD structures were excited by the train of pulses from a mode-locked Ti:sapphire laser after frequency doubling of its output. The laser pulse duration was $\approx 160 \mathrm{fs}$, at a repetition frequency of $\approx 76 \mathrm{MHz}$, with a photon energy of $3.1 \mathrm{eV}(400 \mathrm{~nm})$. The PL signal was detected by a liquid nitrogen cooled Si charge coupled device camera combined with a $0.3 \mathrm{~m}$ focal length single grating monochromator. The streak camera system based on a S20 photocathode was attached to the monochromator, allowing spectral and temporal analysis of the PL signal. The effective time resolution of the setup was $\approx 8$ ps.

In the case of time-correlated single photon counting (TCSPC) experiment the QD structures were excited by 
a picosecond pulsed diode laser PDL $800-\mathrm{B}$ at $3.29 \mathrm{eV}$ $(377 \mathrm{~nm})$, with the pulse duration of $50 \mathrm{ps}$. The PL signal was dispersed by a 0.125 focal length monochromator and detected by a Si-based avalanche photodiode. The photon correlation statistic is built-up by a multichannel picosecond event timer synchronized to the pulse source. The overall setup resolution was around 200 ps.

\section{Experimental results and discussion}

The low temperature PL spectra of investigated ZnTe/ ZnSe QDs are presented in Fig. 1a. The emission bands are spectrally located near $2.0 \mathrm{eV}$ and correspond to the recombination of neutral or charged exciton complexes confined in the dots. The PL band from the QD1 structure is always centered spectrally at the highest energy, whereas the PL emission from QD3 at the lowest one due to the quantum size effect related mainly to hole quantization within the ZnTe island (see sketch in Fig. 1a on top). The apparent asymmetry of the PL bands towards low energy side is the first indication of the staggered band alignment in these dots. It will be further explained in detail.
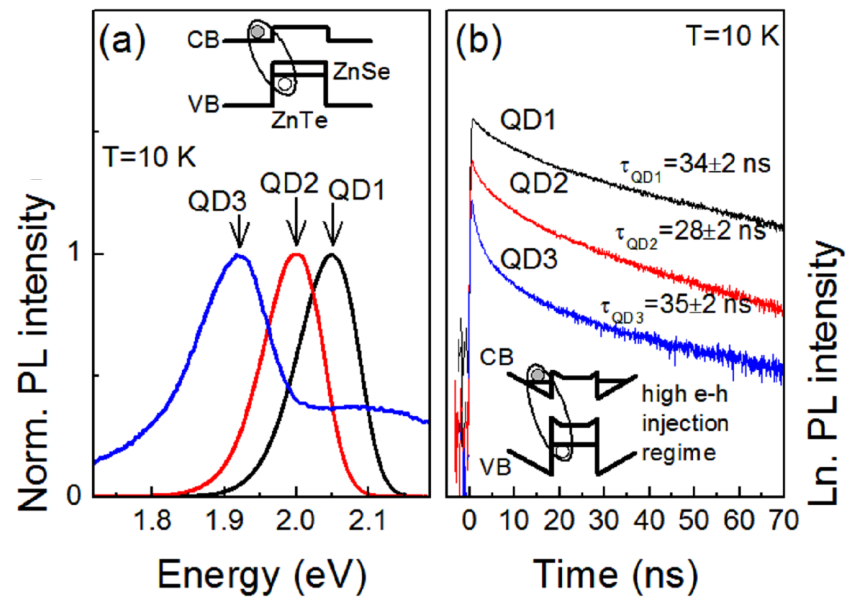

Fig. 1. (a) Low temperature $(T=10 \mathrm{~K})$ photoluminescence spectra of $\mathrm{ZnTe} / \mathrm{ZnSe} \mathrm{QDs}$ of different $\mathrm{ZnTe}$ coverage: 2.5 (QD1), 2.8 (QD2), and 3.0 (QD3) monolayers. A simplified sketch of the QD conduction (CB) and valence band (VB) profiles at the flat-bend conditions is shown on top. (b) PL decay traces measured by the TCSPC technique at the spectral position corresponding to the PL peak maximum for investigated QD structures. An influence of the internal electric field on the QD band structure is schematically depicted in the sketch below.

The type-II confinement for the investigated $\mathrm{ZnTe} /$ ZnSe QDs can be directly resolved within the TRPL experiments $[8,9]$. Figure $1 \mathrm{~b}$ shows TCSPC traces taken at the energy position of the PL peak maximum presented in Fig. 1a. All the traces show a fast non-exponential decay followed by a slow single exponential one. The origin of a fast component is not fully clear. However, it can be related to the photoinjected $\mathrm{e}-\mathrm{h}$ pair recombination under the strong internal electric field across the $\mathrm{ZnTe} / \mathrm{ZnSe}$ heterointerface. The larger is the carrier population, the higher is the strength of the electric field which induces the band-bending near the $\mathrm{ZnTe} / \mathrm{ZnSe}$ heterointerface, as sketched in Fig. 1b [1, 3-6]. The band-bending results in squeezing of the hole wave function towards the $\mathrm{ZnTe}$ / ZnSe heterointerface, but most importantly, it leads to the appearance of a triangle-well-like confinement potential for electrons around the ZnTe island. Consequently, it leads to a stronger spatial localization of an electron and a hole near the $\mathrm{ZnTe} / \mathrm{ZnSe}$ heterointerface area. In contrary, for the flat-band conditions (sketch in Fig. 1a on top) the electron is almost spatially unconfined and therefore the confinement is provided rather by the $\mathrm{e}-\mathrm{h}$ Coulomb interaction. These effects can be directly correlated with the temporal evolution of the PL.

In the first approximation, the radiative recombination of an $\mathrm{e}-\mathrm{h}$ pair depends on the spatial overlap between an electron and hole wavefunctions according to the following $\tau^{-1} \propto \int \psi_{\mathrm{e}} \psi_{\mathrm{h}} \mathrm{d} \boldsymbol{r}$. In the case of a high $\mathrm{e}-\mathrm{h}$ pair injection occurring just after the pump pulse arrival, a relatively strong e-h localization near the $\mathrm{ZnTe} /$ ZnSe heterointerface leads to a significant amplitude of the wave-function overlap integral, and thus to the observation of a short PL decay time. Obviously, for an extended period of time, due to subsequent depopulation of the electron and hole states, the system reaches the flat band condition. Consequently, the PL decay time constant is expected to elongate mostly due to the electron delocalization.

One can note that at high and moderate internal electric field conditions the PL decay cannot be easily parameterized due to unknown recombination statistic, which additionally evolves in time. However, at weak or close to zero internal electric fields, the QD can be viewed as an unperturbed two level system. Therefore, the PL decay can be extracted from a single exponential fit to experimental data. This applied to measured TCSPC traces in Fig. 1b gives the long decay time constants of 34, 28, and $35 \mathrm{~ns}$ for the QD1, QD2, and QD3 structure, respectively. These data are comparable to those existing in the literature for a similar QD system $[4,5]$.

The TRPL experiment based on a streak camera system can give more insight into the dynamic band bending effect, since it provides information concerning the spectral evolution of the overall optical density of states after the laser pulse excitation. Figure $2 \mathrm{a}-\mathrm{c}$ shows an example of the low temperature $(T=10 \mathrm{~K})$ streak camera images after the pulse excitation with an average power of $P=100 \mathrm{~mW}$. Two distinct features are well visible: (i) the PL band shifts toward lower energy side with time, (ii) the PL signal is present at the negative time scale. The first feature is a clear manifestation of already discussed type-II band alignment in the investigated $\mathrm{ZnTe} /$ ZnSe QDs. It also explains the observed asymmetry of the PL emission integrated over time, as seen in Fig. 1a. The second feature indicates incomplete carrier relax- 


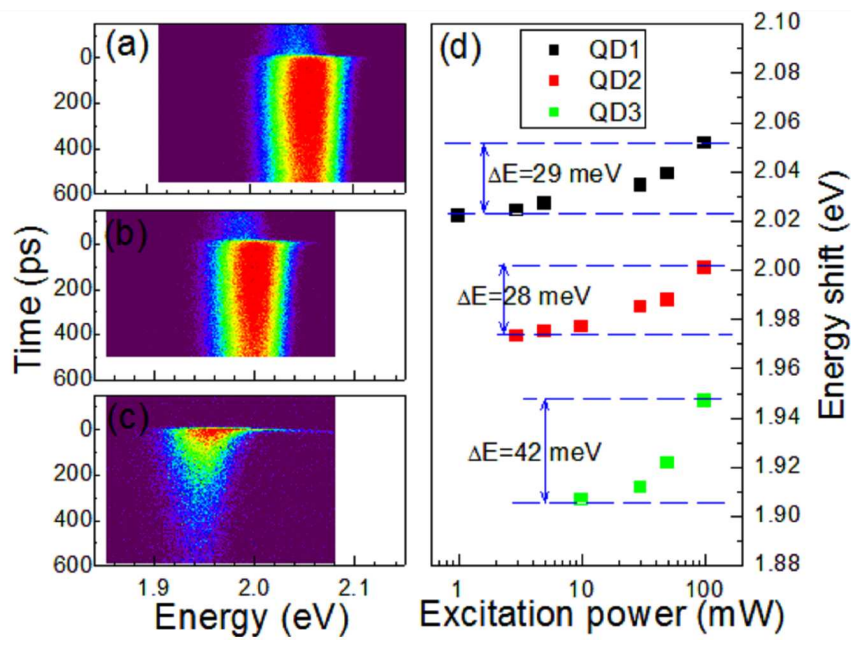

Fig. 2. The time-resolved spectral maps of $\mathrm{ZnTe} / \mathrm{ZnSe}$ QDs emission for QD1 (a), QD2 (b), and QD3 (c) structure measured at $P=100 \mathrm{~mW}$, (d) excitation power dependent spectral shift $\Delta E$ of the PL peak maximum (full squares) related to the dynamic band-bending effect.

ation between two subsequent laser pulses $(\approx 13 \mathrm{~ns}$ in this case).

Thorough analysis of the PL peak energy position shortly before and after the pulse arrival versus the average pump power $P$ can give information about the magnitude of an energy shift $\Delta E$ related to the dynamic band-bending effect. These results are presented in Fig. 2d.

Let us note that $\Delta E$ describes the energy difference between the flat band and the high band-bending condition. One can see that for QD1 and QD2 structures, within the given $P$ range, the band-bending generates $\approx 28 \mathrm{meV}$ energy shift of the PL peak, which is nearly twice smaller than that observed for the QD3 structure $(\approx 48 \mathrm{meV})$. The large difference in $\Delta E$ between QD1, QD2, and QD3 structures can be related to the volume of $\mathrm{ZnTe}$ islands. Due to large size of dots within the QD3 structure, the magnitude of an internal electric field across the $\mathrm{ZnTe} /$ ZnSe heterointerface can be enhanced by more effective collection of $\mathrm{e}-\mathrm{h}$ pairs to the dot after the pulse excitation. This interpretation can be additionally confirmed by registered faster initial PL decay for the QD3 structure in respect of those observed for QD1 or QD2 structure in Fig. 1b.

\section{Conclusions}

The carrier dynamics and dynamic band-bending effect in type-II ZnTe/ZnSe QDs were studied by the time-integrated and time-resolved photoluminescence. The long decay constant corresponding to spatially separated $\mathrm{e}-\mathrm{h}$ pairs under the weak influence of an internal electric field has been obtained and reaches $28^{-}-34$ ns. The time-resolved, excitation power dependent experiment with streak camera detection allows obtaining the magnitude of spectral shift related to the dynamic band bending effect in such QDs. For a given range of excitation powers, the spectral shift is $28 \mathrm{meV}$ for relatively small dots and $42 \mathrm{meV}$ for larger ones.

\section{Acknowledgments}

M.S. acknowledges the financial support from the Iuventus Plus grant no. IP2011 053371, the Foundation for Polish Science through a Subsidy No. 10/2012. The authors acknowledge the financial support of National Council of Taiwan under the project-based personnel exchange program between the NSC and PAS, project No. NSC101-2911-I-011-506.

\section{References}

[1] F. Hiroshi, M. Koji, J. Appl. Phys. 57, 2960 (1985).

[2] P.Y. Yu, M. Cardona, in: Fundamentals of Semiconductors: Physics and Materials Properties, Eds. H.E. Stanley, W.T. Rhodes, 4th ed., Springer-Verlag, Berlin 2010, p. 282.

[3] I.R. Sellers, V.R. Whiteside, I.L. Kuskovsky, A.O. Govorov, B.D. McCombe, Phys. Rev. Lett. 100, 136405 (2008).

[4] T.Y. Lin, D.Y. Lyu, J. Chang, J.L. Shen, W.C. Chou, Appl. Phys. Lett. 88, 121917 (2006).

[5] R. André, R. Najjar, L. Besombes, C. Bougerol, S. Tatarenko, H. Mariette, Phys. Status Solidi C 6, 857 (2009).

[6] V.A. Shuvayev, I.L. Kuskovsky, L.I. Deych, Y. Gu, Y. Gong, G.F. Neumark, M.C. Tamargo, A.A. Lisyansky, Phys. Rev. B 79, 115307 (2009).

[7] C.S. Yang, Y.J. Lai, W.C. Chou, W.K. Chen, M.C. Lee, M.C. Kuo, J. Lee, J.L. Shen, D.J. Jang, Y.C. Cheng, J. Appl. Phys. 97, 033514 (2005).

[8] M. Baranowski, M. Syperek, R. Kudrawiec, J. Misiewicz, J.A. Gupta, X. Wu, R. Wang, Appl. Phys. Lett. 96, 041911 (2010).

[9] M. Baranowski, M. Syperek, R. Kudrawiec, J. Misiewicz, J.A. Gupta, X. Wu, R. Wang, J. Phys., Condens. Matter 24, 185801 (2012). 